\title{
On Calculating the Zeros of Polynomials by the Method of Lucas
}

\author{
Herbert E. Salzer
}

\begin{abstract}
When $f(x)$ is a polynomial of degree $n$ and $x_{i}, i=0,1, \ldots, n$, are any $n+1$ points at which $f\left(x_{i}\right) \neq 0$, the zeros of $f(x)$ are known to be identical with the zeros of ${ }^{1} \Sigma a_{i} /\left(x-x_{i}\right)$, where $a_{i}=f\left(x_{i}\right) / \Pi^{\prime}\left(x_{i}-x_{i}\right)$. Lucas proposed this principle for use in an electric analogue device for finding zeros. The present note evaluates this principle in digital computation for both real and complex zeros when the coefficients of $f(x)$ are given exactly (integral or rational) so that the zeros of $f(x)$ are identical with the zeros of $\Sigma A_{i} /(x-i), A_{\mathrm{i}}$ integral. The chief advantages are (1) the saving of labor in tabulating $\Sigma A_{i} /(x-i)$ instead of $f(x)$ in the neighborhood of the zero, especially for complex zeros, and (2) somewhat less work in the inverse interpolation for the zero. Three examples in locating a real root, and one example in locating a complex root were worked out in support of these findings.
\end{abstract}

In three separate notes F. Lucas ${ }^{2}$ describes an electric analogue device for calculating the roots of equations (also mentioned by J. S. Frame). ${ }^{3}$ Although the principle is familiar, as far as the writer knows it has not been investigated from the standpoint of digital computation. The present note is intended to call attention to its advantages in finding the roots of polynomial equations with exact coefficients when one has a first approximation as a starting point.

If $f(x)$ denotes a polynomial of degree $n$, and $x_{i}$, $i=0,1, \ldots, n$, denotes any $n+1$ points where $f\left(x_{i}\right) \neq 0$, from

$$
\begin{aligned}
f(x) & =\sum \frac{\Pi^{\prime}\left(x-x_{j}\right)}{\Pi^{\prime}\left(x_{i}-x_{\jmath}\right)} f\left(x_{i}\right) \\
& =\Pi\left(x-x_{j}\right) \sum \frac{f\left(x_{i}\right)}{\Pi^{\prime}\left(x_{i}-x_{j}\right)\left(x-x_{i}\right)},
\end{aligned}
$$

there follows the well-known result (which is the basis of Lucas's method) that the zeros of $f(x)$ are identical with the zeros of

$$
\sum \frac{a_{i}}{x-x_{i}}, \text { where } a_{i}=\frac{f\left(x_{i}\right)}{\Pi^{\prime}\left(x_{i}-x_{j}\right)} \text {. }
$$

In problems where the coefficients of $f(x)$ are rational, the choice of $x_{i}=i$ is very convenient. By multiplying through, one obtains the equation in the form $\Sigma A_{i} /(x-i)=0, A_{i}$ integral, which saves a considerable number of multiplication operations, especially when getting all or even several of the zeros of the same $f(x)$.

By choosing $x_{i}=x_{0}+i h$, for any $x_{0}$ and $h$ for which $f\left(x_{i}\right) \neq 0$, Lucas's principle can be formulated also as follows:

The zeros of $f(x)$, a polynomial of the $n$th degree, are identical with the zeros of $\Delta^{m}\{f(t) /(x-t)\}, m$

${ }^{1}$ Here and elsewhere the summation is over the range 0 to $n$; similarly $\Pi$ indicates a product over the same range, and $\Pi^{\prime}$ indicates such a product with the vanishing factor omitted.

${ }^{2}$ Lueas, Compt. rend. Acad. Sci. Paris 106, 645-48 and 1072-74 (1888); 111, 965-67 (1890).

${ }^{3}$ J. S. Frame, MTA C 1, 337-53 (especially 347-50) (1945). any integer $\geq n$, where the $f(t) /(x-t)$ is tabulated for any $m+1$ equally spaced values of $t$.

Of course, in obtaining $\Delta^{m}\{f(t) /(x-t)\}, t$ is the variable, with $x$ as the parameter, and the resulting expression is then regarded as a function of $x$. (The proof is left for the reader.)

This principle of Lucas, namely, calculating the zeros of $f(x)$ by tabulating $\Sigma a_{i} /\left(x-x_{i}\right)$ or $\Sigma A_{i} /(x-x)_{i}$, instead of $f(x)$ itself, in the neighborhood of a zero, was tried out in several different examples where the zeros had already been obtained from the tabulation of $f(x)$ itself. In all examples, the use of Lucas's method showed a very great saving of computational labor. The two main advantages were (1) much less work in calculating $A_{i} /\left(x-x_{i}\right)$ instead of the separate terms of $f(x)$, especially those of high degree, ${ }^{4}$ and (2) somewhat less work in the inverse interpolation from the tabulated $\Sigma A_{i} /\left(x-x_{i}\right)$ instead of the tabulated $f(x)$ near the zero, which was apparent from the tendency of $\Delta^{m} / \Delta$ to be less in the former case. ${ }^{5}$

In connection with (1), in adding the separate terms of $\Sigma A_{i} /\left(x-x_{i}\right)$ considerably more significant figures were lost than in the summation of the separate terms of $f(x)$; but that disadvantage is slight because of the ease in getting any number of places in $A_{i} /\left(x-x_{i}\right)$ by performing continued division on an ordinary 10 -bank desk calculator (the $x-x_{i}$ is almost certainly an exact number having fewer than 10 significant figures), and the total work in tabulating the $\Sigma A_{i} /\left(x-x_{i}\right)$ is still much less than that in tabulating the $f(x)$. But if the $x_{i}$ 's are not exact, or the coefficients in $f(x)$ are approximate, this principle of Lucas is severely limited in applicability, even to the point of not yielding a single significant figure. Thus, if in the example below, one were to introduce a relative error of $10^{-10}$ in those exact coefficients of $f(X)$, it is apparent that the $h(X)$ could not be obtained to even one significant figure. However, the choices of $x_{i}=i$ or $x_{i}=i-[n / 2]$ seem most suitable for many problems. For example, in the case of the classical

4 There is the very well-known computational scheme for $a_{n} x^{n}+a_{n-1} x^{n-1}+$ $a_{n-2} x^{n-2}+\ldots+a_{1} x+a_{0}$ in the form $u_{n}=a_{n} x+a_{n-1}, u_{n-1}=u_{n} x+a_{n-2}, \ldots$, ete. until $f(x)=u_{1}=u_{2} x+a_{0}$, which avoids the calculation of powers of $x$, but which may be less convenient for checking and the retention of significant figures.

${ }_{5}$ The rate of convergence of most inverse interpolation series depends upon the rapidity with which the $\Delta^{m} / \Delta$ fall off. 
orthogonal polynomials where the coefficients are given exactly (for example, Hermite, Laguerre, Chebyshev, Legendre, etc.) the $A_{i} /\left(x-x_{i}\right)$ can be easily had to any required number of places.

As an illustration, consider the calculation of the zero of the polynomial

$$
\begin{aligned}
f(X)= & X^{6}-156 X^{5}+8580 X^{4}-205920 X^{3} \\
& +2162160 X^{2}-8648640 X+8648640,
\end{aligned}
$$

which is near 67.28. Here $f(X)$ was chosen to be $H_{13}(x) /(2 x)$, where $X=4 x^{2}$ and $H_{13}(x)$ is the Hermite polynomial of order 13 . Choosing $X_{i}=i$, one has

$$
\begin{aligned}
& f(0)=8648640 \quad f(4)=-2489408 \\
& f(1)=1964665 \quad f(5)=-1389935 \\
& f(2)=-1515008 \quad f(6)=\quad 69120 \\
& f(3)=-2739879
\end{aligned}
$$

The numbers $\Pi^{\prime}\left(X_{i}-X_{j}\right)$ have no more than three digits. After obtaining the $a_{i}$ in their lowest terms, one sees that the zeros of $f(X)$ are identical with the zeros of

$$
\begin{aligned}
g(X) \equiv & \frac{12012}{X}-\frac{392933}{24(X-1)}-\frac{94688}{3(X-2)}+\frac{304431}{4(X-3)} \\
& -\frac{155588}{3(X-4)}+\frac{277987}{24(X-5)}+\frac{96}{X-6}
\end{aligned}
$$

\begin{tabular}{|c|c|c|c|c|}
\hline & $X=67.2838$ & $X=67.2839$ & $X=67.2840$ & $X=67.2841$ \\
\hline $288288 / X$ & 4284. 6569307916615887 & 4284. 6505627646435477 & 4284. 6441947565543071 & 4284. 6378267673937825 \\
\hline$-392933 /(X-1)=$ & -5928.0397321819207710 & -5928.0307887737444538 & -5928.0218453925532557 & -5928.0129020383470546 \\
\hline$-757504 /(X-2)=$ & -11603.2461345693724936 & -11603.2283610507337950 & -11603.2105875865449421 & -11603.1928141768056847 \\
\hline $1826586 /(X-3)=$ & 28414. 4061178710654940 & 28414. 3619164363083136 & 28414.31771 51390 70375 2 & 28414. 2735139793510370 \\
\hline$-1244704 /(X-4)=$ & -19668.6039713165138629 & -19668.5728913673145934 & -19668.5418115163390430 & -19668.5107317635867461 \\
\hline $277987 /(X-5)=$ & 4463.2312093995549405 & 4463. 2240434526418545 & 4463. 2168775287393231 & 4463. 2097116278472355 \\
\hline $2304 /(X-6)=$ & 37. 5955799085565843 & 37.5955185619714150 & 37. 5954572155864500 & 37. 5953958694016882 \\
\hline$h(X)=$ & -0.0000000969685200 & +0.0000000237722886 & +0.0000001445132146 & +0.0000002652542578 \\
\hline
\end{tabular}

which, in turn, are identical with the zeros of

$$
\begin{aligned}
24 g(X) \equiv & h(X) \equiv \frac{288288}{X}-\frac{392933}{X-1}-\frac{757504}{X-2} \\
& +\frac{1826586}{X-3}-\frac{1244704}{X-4}+\frac{277987}{X-5}+\frac{2304}{X-6}
\end{aligned}
$$

An approximate value of the zero is $X=67.2838$, and $h(X)$ was calculated for $X=67.2838(0.0001) 67.2841$. The separate terms of $h(X)$ are given here to show the loss in significant figures upon summation:
That only three values of $h(X)$ at intervals of 0.0001 are required in order to find the zero $\bar{X}$ to the maximum attainable accuracy, follows from these differences:

\begin{tabular}{|c|c|c|c|}
\hline$X$ & $h(X)$ & $\Delta$ & $\Delta^{2}$ \\
\hline 67.2838 & $-0 .(6) 0969685200$ & $+0 .(6) 1207408086$ & $+0 .(12) 1174$ \\
67.2839 &.$+(6) 0237722886$ &.$(6) 1207409260$ &.$(12) 1172$ \\
67.2840 &.$(6) 1445132146$ &.$(6) 1207410432$ & \\
67.2841 &.$(6) 2652542578$ & $\ldots \ldots$ & \\
\hline
\end{tabular}

When these differences were compared with the corresponding differences in $f(X)$, it was noted that $\Delta^{2} / \Delta$ for $h(X)$ was only $(1 / 25)$ of $\Delta^{2} / \Delta$ for $f(X)$, from which one can infer that the inverse interpolation is better for the function $h(X)$. The zero $\bar{X}=67.2839+0.0001 p$ was found from the threepoint formula ${ }^{6} p=r-r^{2} s$, where

Thus

$$
\begin{aligned}
& r=-2 h(67.2839) /\{h(67.2840)-h(67.2838)\} \\
& s=\Delta^{2} /\{h(67.2840)-h(67.2838)\} .
\end{aligned}
$$

$$
h(67.2840)-h(67.2838)=0 .(6) 2414817346,
$$

${ }^{6}$ H. E. Salzer, Bul. Am. Math. Soc. 50, No. 8, 513-16 (1944).

$$
\begin{aligned}
r & =-0 .(7) 475445772 / 0 .(6) 2414817346 \\
& =-0.196886846, \\
s & =0 .(12) 1174 / 0 .(6) 24148=0 .(6) 486, \\
r^{2} & =0.03876, r^{2} s=0 .(7) 1884, \\
p & =-0.196886865 ; \bar{X}=67.2838803113135,
\end{aligned}
$$

which happens to be correct to 13 decimals.

This method was tested upon the calculation of two different roots of the same tenth-degree polynomial in $X$ (the example was to find both the smallest and largest zero of $H_{20}(x)$ ), the result being that the relative saving of labor was even greater than that for $H_{13}(x)$. In fact, as the degree of the polynomial increases, the proportion of work saved also increases.

Finally, this method was applied to the computation of a complex root of a tenth-degree polynomial, from a rough first approximation. There the relative saving of labor was even greater than that for the examples involving real roots, due in particular to the avoidance of the calculation of high powers of complex numbers. Even when the computation is for complex roots, the choice of $x_{i}=i$ or $x_{i}=i-[n / 2]$ is still suitable, so that if the coefficients of $f(x)$ are real, the calculation involves the sum of fractions with only real numerators.

Washington, July 24, 1951. 